\title{
COMPOSITE COATINGS CONTAINING NANOMETRIC CONSTITUENTS PREPARED BY PLASMA SPRAYING WITH LIQUID FEEDSTOCKS
}

\author{
Radek MUSALEK, Tomas TESAR, Jan MEDRICKY, Jan CIZEK, Frantisek LUKAC \\ Institute of Plasma Physics of the Czech Academy of Sciences, Prague, Czech Republic, EU, \\ musalek@ipp.cas.cz
}

https://doi.org/10.37904/nanocon.2021.4310

\begin{abstract}
Plasma spraying of liquid feedstocks allows deposition of materials with novel microstructures. Due to the combination of a liquid carrier and ultrafine powders (in case of suspensions) or in-situ formation of the final material in the plasma jet (in case of solutions), preparation of ultrafine composites is possible, typically in a form of coatings on the substrates. Moreover, both suspension and solution spraying routes can be easily combined, either together or even with conventional plasma spraying of coarse dry powders, thus merging benefits of "nanometric" and "micrometric" constituents in the final composite microstructure. As a consequence, liquid feedstock plasma spraying represents an industrially-relevant deposition process with wide variability, providing coatings with properties tailored for various applications ranging from compact wearresistant layers, porous thermal barrier coatings, bio-compatible medical coatings, catalyst carriers, fuel-cells, etc. The paper introduces several examples of such ultrafine coatings recently deposited at IPP CAS using hybrid water-stabilized plasma torch.
\end{abstract}

Keywords: Plasma spraying, liquid feedstock, coatings, functionally graded materials (FGMs)

\section{INTRODUCTION}

Conventional plasma spraying is based on spraying of relatively coarse dry powders, with typical size in tens of micrometers [1]. Decreasing particle size is needed to achieve finer coatings microstructures. However, too small particles do not possess high enough momentum to penetrate the plasma jet and get heat-treated properly and thus provide poor deposition efficiency. Small dry particles may even get entirely evaporated in the plasma jet. These problems may be solved by plasma spraying of liquid feedstocks allowing deposition of coatings from suspensions and solutions [2]. Due to the combination of a liquid carrier and ultrafine powders (suspensions route) or in-situ formation of the final material in the plasma jet (solutions route), preparation of ultrafine composites is possible, as the liquid carrier provides the needed momentum and the injected material can thus penetrate the plasma jet. However, as the primary stream of the liquid gets fragmented in the plasma due to the immense shear forces, each created droplet contains multiple primary nanoparticles, which agglomerate after the liquid carrier evaporation. It has therefore turned out, that introduction of nanometric features into the microstructure may be quite challenging as most of the primary particles agglomerate during the in-flight stage [3]. However, the deposited droplets are still significantly smaller (particularly in terms of volume) than in the case of conventional spraying of dry powders, enabling formation of features with size of several tens of nanometers. Also, nanometric features may be present in the coating in the form of ultrafine porosity, untreated primary particles (e.g., particles which were deliberately not entirely melted), condensates (i.e. material which was evaporated but condensed again on the coated part-sample) or from solution.

This paper introduces examples of several coatings prepared recently at the Institute of Plasma Physics of the Czech Academy of Sciences having submicron-sized features and deposited with different geometries, 
including relatively homogeneous single-layers, heterogeneous multilayers, intermixed coatings and functionally graded coatings (FGM).

\section{EXPERIMENTALS}

All coatings shown in this study were deposited by hybrid water/argon stabilized plasma torch WSP-H 500 (ProjectSoft HK a.s., Czech Republic) with typical operation power $150 \mathrm{~kW}$. The deposition took place in the open air atmosphere onto the substrates attached to a revolving carousel. The substrates were first grit-blasted by alumina, residues of which may be found on the substrate-coating interface. Injection of the liquid feedstocks into plasma was radial with typical injection distance from the plasma nozzle in the range of 20 to $30 \mathrm{~mm}$. When spraying liquid feedstocks, typical stand-off distance of the plasma torch from the coated substrates was $100 \mathrm{~mm}$, which guaranteed proper heat treatment of the fragmented particles along their whole trajectory in the plasma jet (typical jet length was also $\sim 100 \mathrm{~mm}$ ) without losing too much velocity, ensuring high deposition efficiency. Due to the immense heat transfer from the plasma towards the samples, these were cooled by compressed air. The plasma torch was mounted on programmable robotic arm allowing deposition by consecutive strokes of the torch in front of the carousel separated by cooling periods. Liquid feedstocks were injected into plasma with very high feed rates, typically around $100 \mathrm{ml} / \mathrm{min}$. More details on the deposition with WSP-H are provided e.g. in [4,5] or papers referenced for each coating example.

Shadowgraphy of the feedstock injection into plasma jet was carried out by SprayCam imaging system (Control Vision Inc., USA). Metallographic cross-sections and free-surfaces of the deposited coatings were investigated by EVO MA 15 (Carl Zeiss, Germany) scanning electron microscope in back-scattered electron detection mode.

\section{RESULTS}

\subsection{Coating Formation}
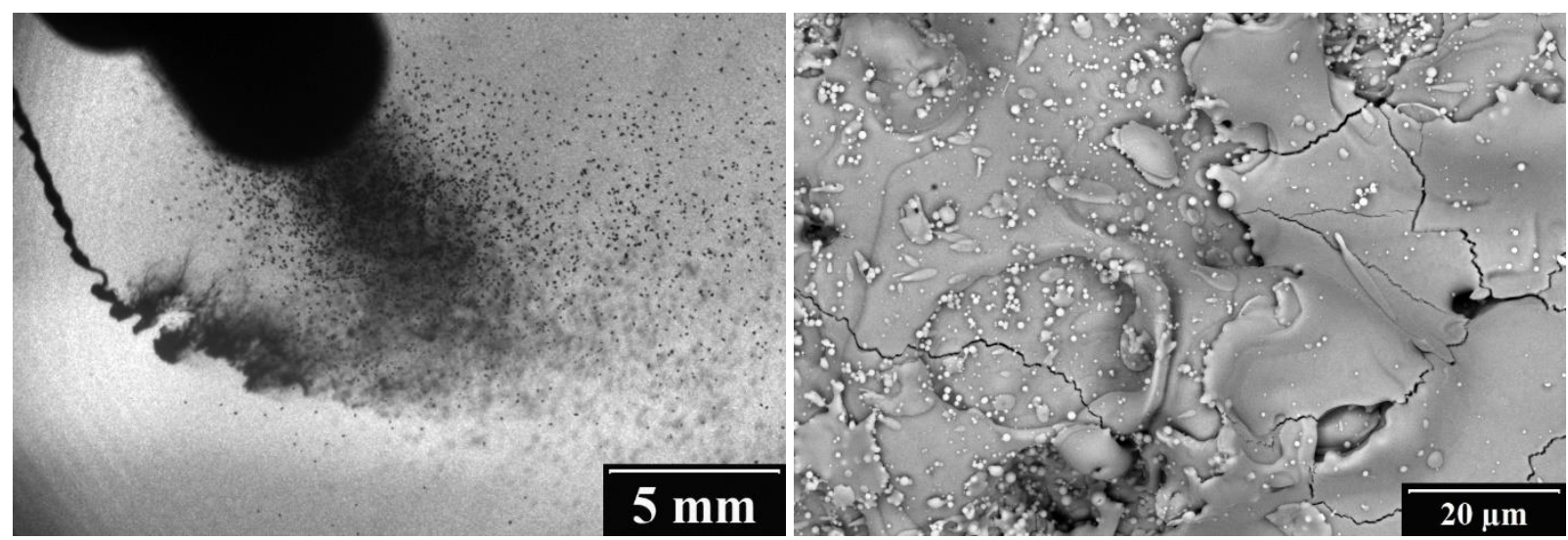

Figure 1 Left: Shadowgraphy of concurrent injection of liquid stream and coarse dry powder into the plasma jet during so-called hybrid plasma spraying. Right: Illustration of the hybrid YSZ coating surface incorporating both conventional large splats from coarse dry powder and miniature splats deposited from suspension.

Both main setups of thermal spraying using conventional dry powder or liquid feedstocks may be illustrated in Figure 1. In the left figure, shadowgraphy of the injection point is depicted. In this so-called hybrid spraying setup [6], continuous liquid stream is fragmented in the plasma jet into smaller droplets which are further downstream accompanied by coarser dry particles. Subsequently, all droplets are dragged towards the coated part while the liquid carrier is evaporated and the solid phase(s) melted. After the impact of molten droplets on 
the substrate, material is deposited in form of flattened lamellae - splats (Figure 1 - right). These are relatively big when originated from the coarse dry powders, having characteristic diameter of several tens of micrometers. In case of ceramics, their cracking is common, due to the evolution of quenching stresses exceeding their relatively low local strength and limited ability to deform plastically [7]. Nevertheless, these cracks may be welcome as they improve for example thermal shock resistance or strain tolerance of the deposited ceramic layers. On the other hand, splats originating from liquids are much smaller which may provide other benefits if needed, such as suppressed formation of the intrasplat cracks. Also, due to their rather lower momentum at the time of impact, they often tend to retain their round shape even when deposited (Figure 2 - right). In case of plasma spraying with liquid feedstocks only, the deposited coatings may consist solely of such submicron-sized particles. Naturally, also the porosity within the coating becomes much finer when compared to the conventional coatings sprayed from coarse powders.

\subsection{Coatings with Ultrafine Features}

It is a great benefit of thermal spraying that different layers may be easily combined e.g. by alternating feedstocks in the feedline. Example of such layered coating is shown in Figure 2, where a triple layer consisting of metallic bond-coat was overlaid by yttria stabilized zirconia (YSZ) deposited first from dry coarse powder and consequently from ethanol-based YSZ suspension [8]. A magnified detail of interface between ceramic sublayers (Figure 2-right) illustrates a substantial difference in the size of achievable microstructural features, including sub-micron sized lamellae and pores.
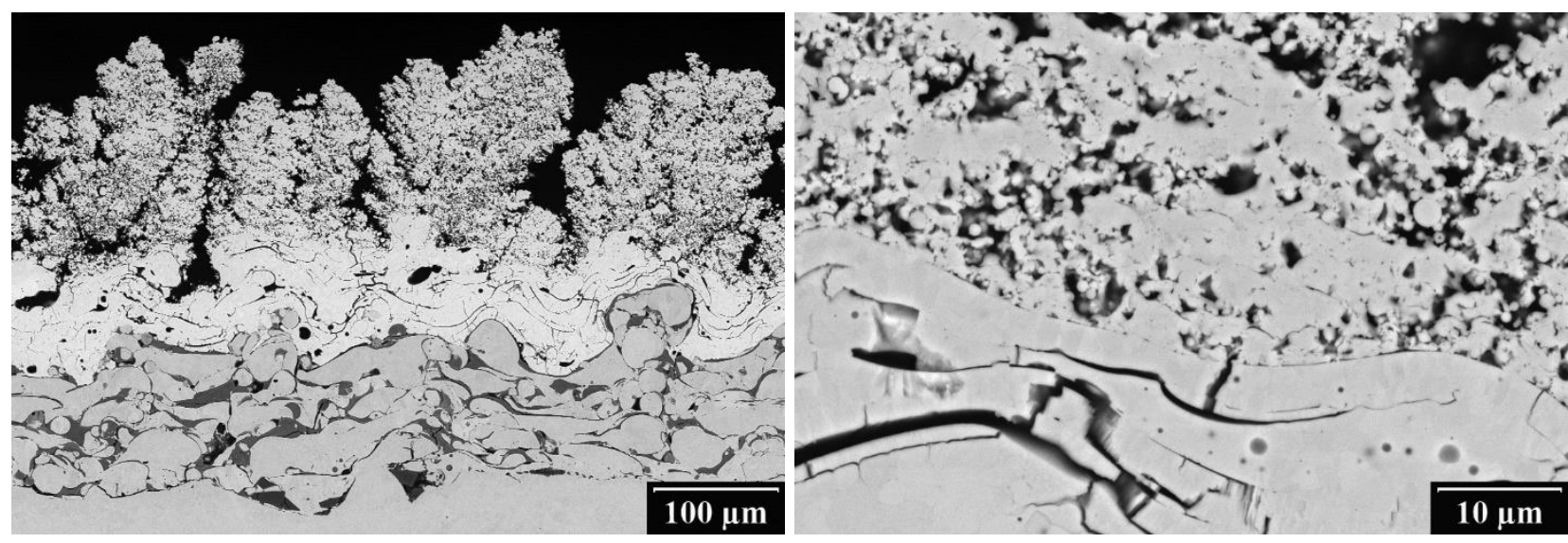

Figure 2 Layered coating consisting of NiCrAlY bond-coat (at the bottom) oversprayed by coarse YSZ from powder and columnar ultrafine YSZ deposited from suspension
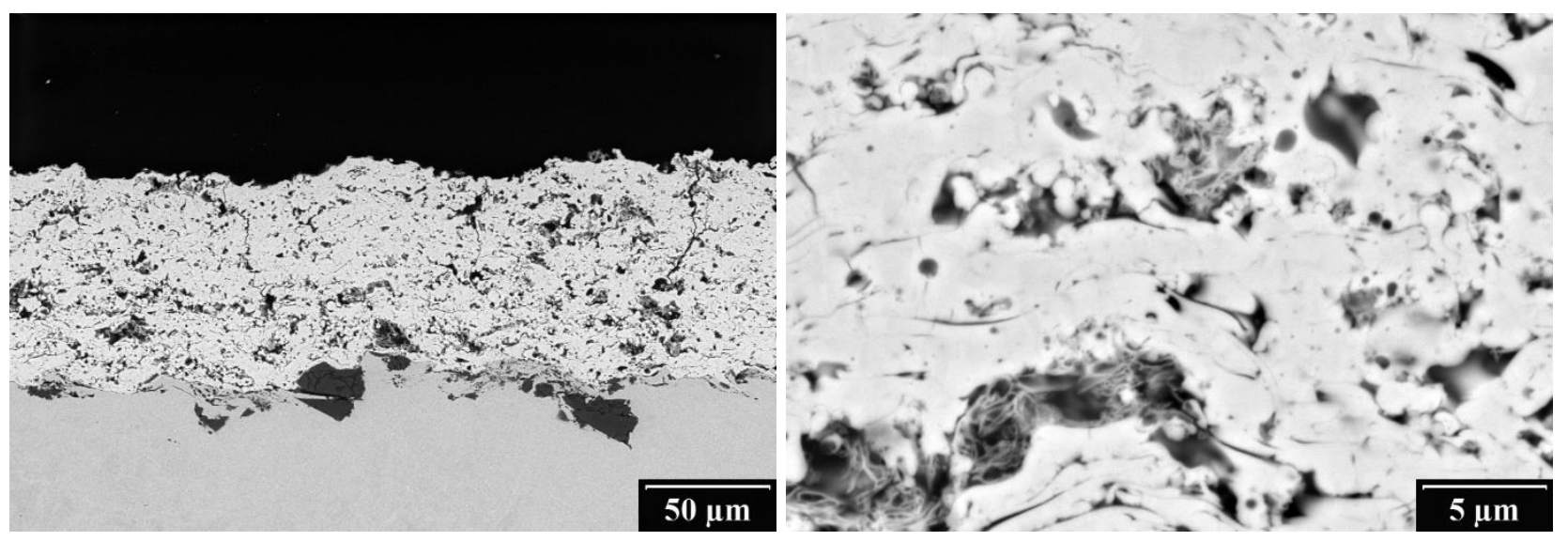

Figure 3 Vertically cracked YSZ ceramic deposited from solution 
Aside from the size of the features, also their overall morphology may be altered. Figure 3 shows a typical microstructure of a vertically cracked YSZ ceramics deposited from solution (mixture of yttrium nitrate hexahydrate + zirconium acetate in dilute acetic acid) [4]. Areas of rather dense coating intermingled with highly porous thin-walled "foamy" areas may be observed. A much more porous alumina coating was deposited from aqueous solution of aluminium nitrate nonahydrate (Figure 4) [9]. Extremely porous ultrafine coating, which was nevertheless quite fragile and thin (Figure 5), was deposited from solution of titanium isopropoxide in ethanol [10]. In this case, a "tree-like" morphology of the coating suggests that the coating was predominantly formed by condensation of a vaporized feedstock rather than conventional deposition of the molten droplets.
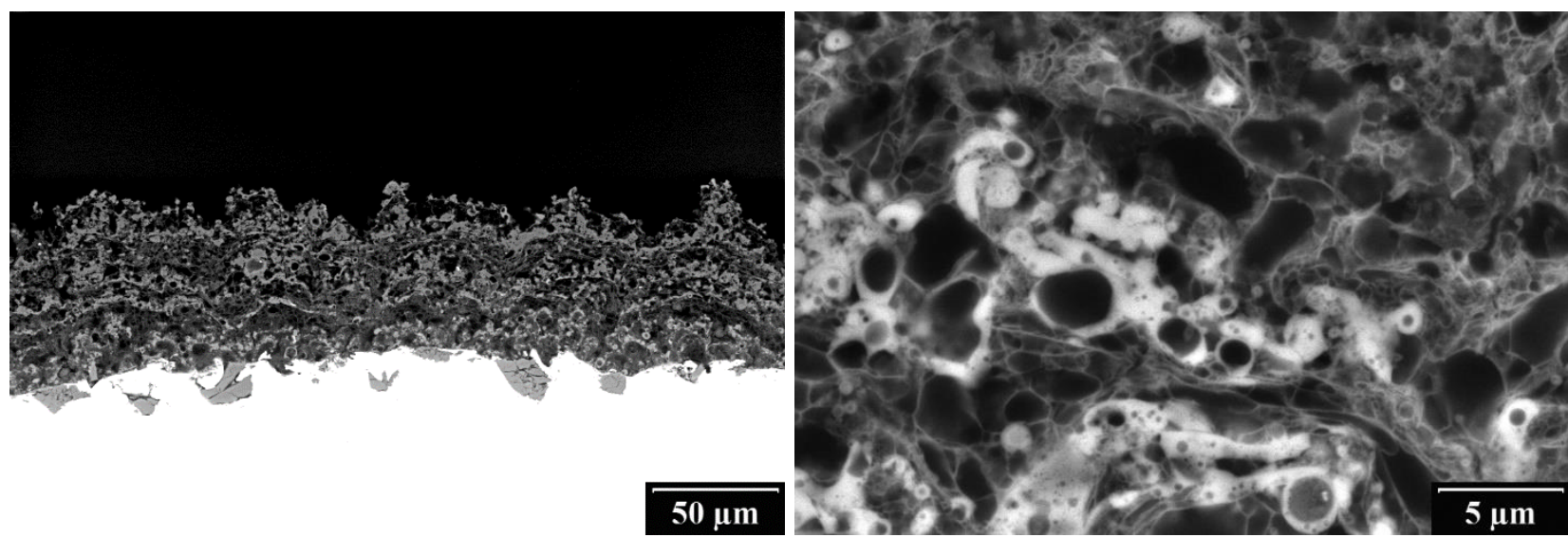

Figure 4 Porous alumina ceramic deposited from solution
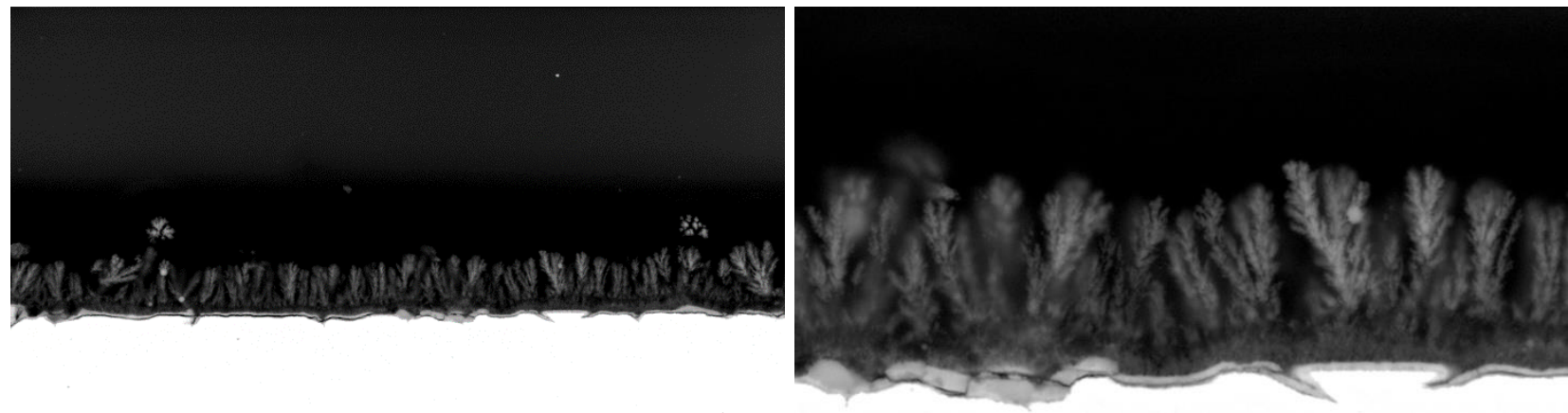

$20 \mu \mathrm{m}$

Figure 5 Ultra-fine titania condensed during spraying of solution of titanium isopropoxide

\subsection{Intermixed Coatings}

Another possibility to achieve novel ultrafine microstructures by thermal spraying is deposition of composite coatings. Figure 6 illustrates such coating deposited from aqueous hydroxyapatite suspension [11]. Two types of features may be observed within the coating; rather dense flattened splats with characteristic diameter around 10 micrometers and much finer droplets originating from the feedstock that was not thoroughly processed, i.e. where the liquid carrier was evaporated but the primary particles were deposited without considerable agglomeration. In the following experiments (Figure 7), a composite coating was deposited also by using aqueous hydroxyapatite (HAp) suspension as feedstock. However, this time, with addition of aqueous silver nitrate solution, which resulted into incorporation of homogeneously dispersed Ag nanoparticles within the coating. Integration of such submicron-features may be beneficial e.g. for improvement of antibacterial properties of the HAp coatings (currently under survey). 


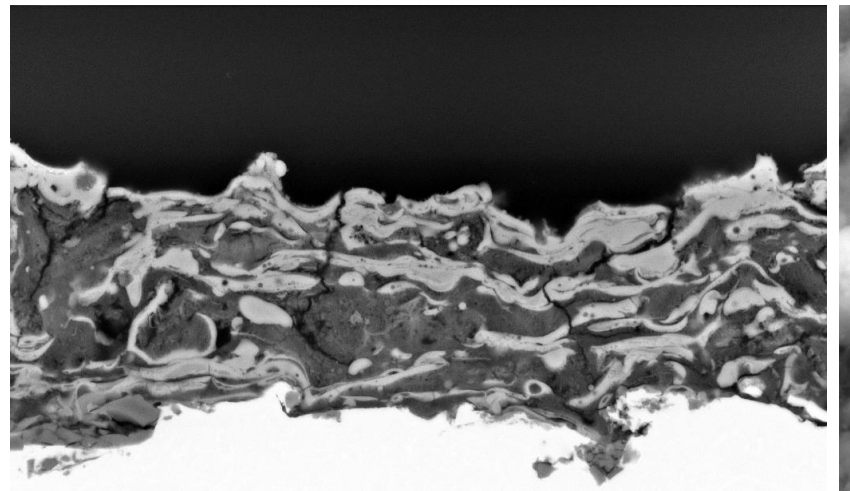

$20 \mu \mathrm{m}$

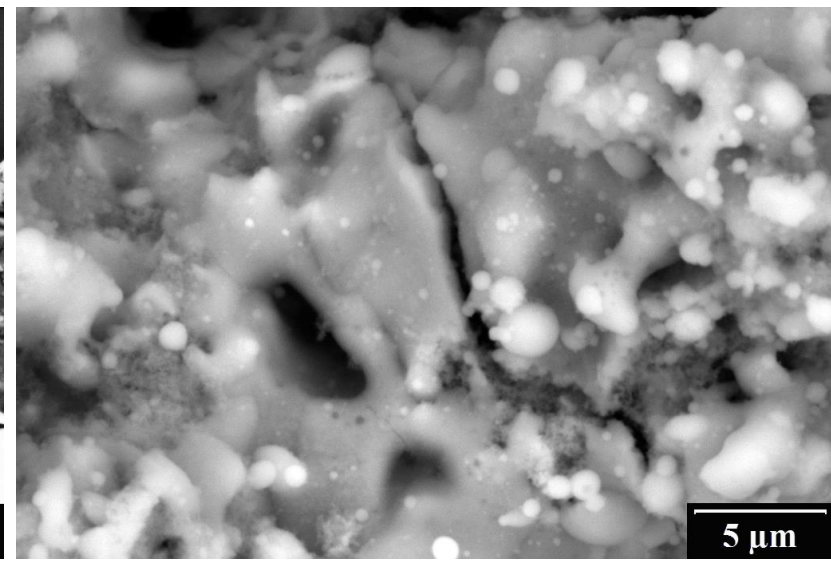

Figure 6 Hydroxyapatite coating. Cross-section (left) and free-surface (right) showing submicron features.
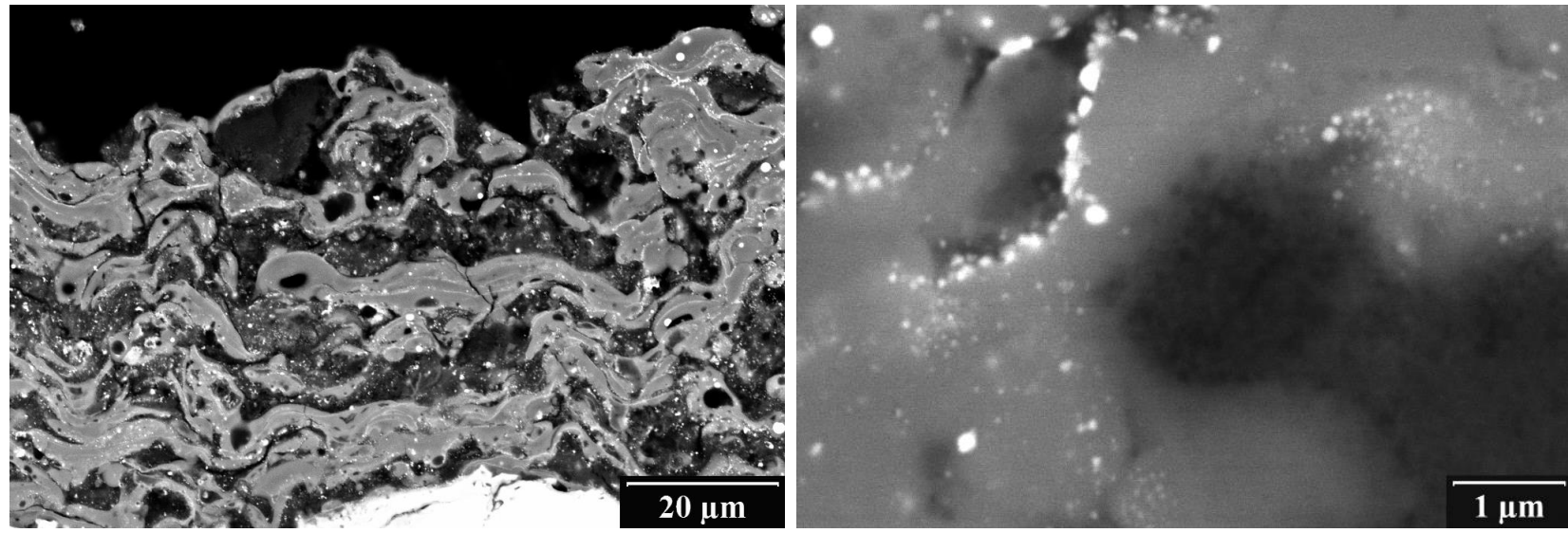

Figure 7 Hydroxyapatite coating with homogenously dispersed antibacterial Ag nanoparticles (brightest phase)

\subsection{GRADIENT COATINGS}

Recent modifications to the liquid injection unit enabled continuous change of the feedstock composition, for example, from suspension A to suspension B. In this way, a novel coating with gradual change from alumina to YSZ was deposited (Figure 8). Such microstructure may potentially provide substantial functional benefits such as mitigation of stresses evolving during thermal loading on the sharp interfaces of conventional layered coatings.
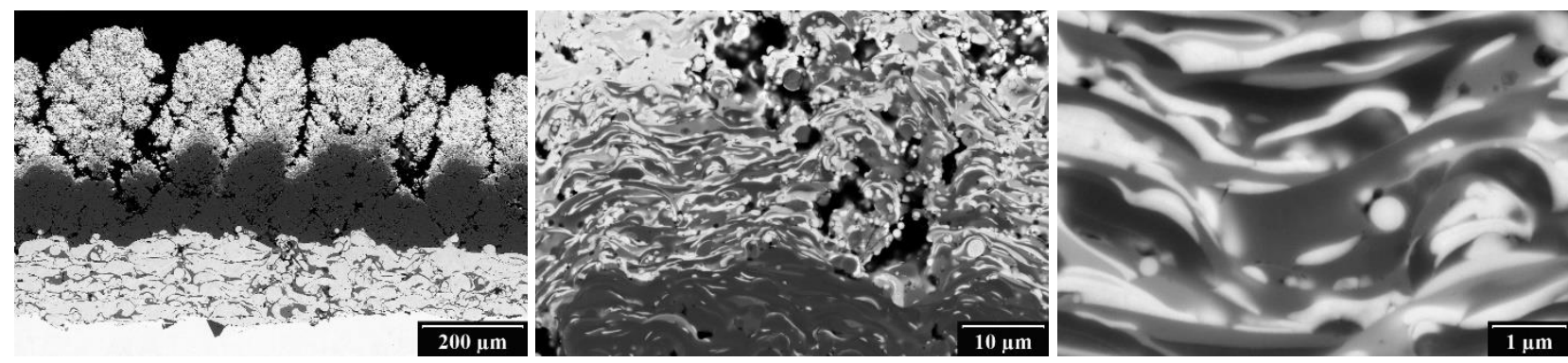

Figure 8 Microstructure of multilayer coating with functionally graded interface between alumina (dark) and yttria-stabilized zirconia (bright). Ceramic coatings were deposited over NiCrAlY bond-coat. 


\section{CONCLUSION}

As illustrated in the selected examples, recent advances in plasma spraying with liquid feedstocks enabled deposition of novel coatings with interesting sub-micron/nanometric sized features. These may be reflected in improved functional properties of the deposits when compared to conventional coatings or may be used in novel applications such as catalysis, SOFCs, etc., where ultrafine composite coatings/layers are needed. Due to the high feed rates achievable with WSP-H technology, plasma spraying with liquid feedstocks may be also both cost- and time-efficient. For example, deposition of application-relevant coating thickness (typically several tens to several hundreds of micrometers) onto 20 test coupons $(20 \times 30 \mathrm{~mm})$ usually takes just several minutes. The ability of the developed method to coat large areas in the open-air atmosphere (i.e., without costly and space-constrained chamber providing vacuum or protective gas atmosphere) and high efficiency of the deposition process makes it an ideal candidate for deposition of such coatings even at industrial scale.

\section{ACKNOWLEDGEMENTS}

Financial support through project 19-10246S "Deposition mechanism and properties of multiphase plasma sprayed coatings prepared with liquid feedstocks" funded by Czech Science Foundation is gratefully acknowledged.

\section{REFERENCES}

[1] DAVIS, J.R. Handbook of Thermal Spray Technology. Davis J.R., ed. Materials Park, OH, USA: ASM International, 2004.

[2] FAUCHAIS, P., JOULIA, A., GOUTIER, S. et al. Suspension and solution plasma spraying. Journal of Physics D: Applied Physics. 2013, vol. 46, pp. 224015.

[3] FAUChAIS, P., VARDELLE, M., GOUTIER, S., VARDELLE, A.. Key Challenges and Opportunities in Suspension and Solution Plasma Spraying. Plasma Chemistry and Plasma Processing. 2014, vol. 35, pp. 511-25.

[4] MUSALEK, R., MEDRICKY, J., TESAR, T. et al. Controlling Microstructure of Yttria-Stabilized Zirconia Prepared from Suspensions and Solutions by Plasma Spraying with High Feed Rates. Journal of Thermal Spray Technology. 2017, vol. 26, pp. 1787-803.

[5] MUSALEK, R., MEDRICKY, J., TESAR, T. et al. Suspensions Plasma Spraying of Ceramics with Hybrid WaterStabilized Plasma Technology. Journal of Thermal Spray Technology. 2017, vol. 26, pp. 37-46.

[6] JOSHI, S.V., SIVAKUMAR, G. Hybrid Processing with Powders and Solutions: A Novel Approach to Deposit Composite Coatings. Journal of Thermal Spray Technology. 2015 vol. 24, pp. 1166-86.

[7] KURODA, S., CLYNE, T.W. The quenching stress in thermally sprayed coatings. Thin Solid Films. 1991, vol. 200, pp. 49-66.

[8] TESAR, T., MUSALEK, R., MEDRICKY, J., CIZEK, J., LUKAC, F., CHRASKA, T. Deposition of multiphase coatings from liquid feedstock using hybrid water-stabilized plasma torch. In: ITSC 2018 - Proceedings of the International Thermal Spray Conference. Orlando: ASM International, 2018, pp. 456-62.

[9] TESAR, T., MUSALEK, R., LUKAC, F. et al. Solution Precursor Plasma Spraying of Cr-Doped $\mathrm{Al}_{2} \mathrm{O}_{3}$ Thermochromic Coatings. Journal of Thermal Spray Technology. 2019, vol. 29, pp. 199-211.

[10] MUSALEK, R., MEDRICKY, J., TESAR, T., KOTLAN, J., LUKAC, F. Deposition of titania from solution by hybrid water-stabilized plasma torch. In: METAL 2016 - Conference Proceedings: 25th Anniversary International Conference on Metallurgy and Materials. Brno: Tanger, 2016, pp. 1121-6.

[11] CIZEK, J., BROZEK, V., CHRASKA, T. et al. Silver-Doped Hydroxyapatite Coatings Deposited by Suspension Plasma Spraying. Journal of Thermal Spray Technology. 2018, vol. 27, pp. 1333-43. 